\begin{tabular}{|c|l|}
\hline Title & Formation of a Pd atomic chain in a hydrogen atmosphere \\
\hline Author(s) & Kiguchi, Manabu; Hashimoto, Kunio; Ono, Y uriko; Taketsugu, Tetsuya; Murakoshi, Kei \\
\hline Citation & $\begin{array}{l}\text { Physical Review B, 81(19), 195401 } \\
\text { https://doi.org/10.1103/PhysRevB.81.195401 }\end{array}$ \\
\hline Issue Date & 2010-05-15 \\
\hline Doc URL & http://hdl.handle.net/2115/47801 \\
\hline Rights & ○2010 The A merican Physical Society \\
\hline Type & article \\
\hline File Information & PRB81-19_195401.pdf \\
\hline
\end{tabular}

Instructions for use 


\title{
Formation of a Pd atomic chain in a hydrogen atmosphere
}

\author{
Manabu Kiguchi, * Kunio Hashimoto, Yuriko Ono, Tetsuya Taketsugu, and Kei Murakoshi \\ Division of Chemistry, Graduate School of Science, Hokkaido University, N10W8 Kita, Sapporo 060-0810, Japan
}

(Received 3 March 2010; revised manuscript received 2 April 2010; published 3 May 2010)

\begin{abstract}
The formation of a $\mathrm{Pd}$ atomic chain in a hydrogen atmosphere was investigated by measurements of conductance and vibrational spectroscopy of a single molecular junction and the theoretical calculation. While atomic chains were not formed for clean $3 d$ and $4 d$ metals, in the case of Pd (a $4 d$ metal) atomic chains could be formed in the presence of hydrogen. Stable atomic chains with two different atomic configurations were formed when the Pd atomic contact was stretched in a $\mathrm{H}_{2}$ atmosphere: highly conductive short hydrogenadsorbed atomic chain and low conductive long hydrogen incorporated atomic chain.
\end{abstract}

DOI: 10.1103/PhysRevB.81.195401

PACS number(s): 68.65.-k, 73.40.Cg, 73.40.Jn, 73.63.Rt

\section{INTRODUCTION}

Chains of single metal atoms forming bridges between metal electrodes have attracted wide attention due to their unique properties ${ }^{1}$ and their potential applications as ultimate conducting wires in nanoscale electronic devices. ${ }^{2}$ In the case of $\mathrm{Au}$, atomic chains up to $2 \mathrm{~nm}$ long have been created in UHV and their physical properties have been investigated with various techniques. ${ }^{2-4}$ The formation of atomic chains was also reported for other $5 d$ metals such as Pt and Ir. On the other hand, it has not been possible to prepare atomic chains in the case of $3 d$ and $4 d$ metals for the following reasons. ${ }^{2,5}$ The formation of the atomic chain requires relatively strong metal-metal bonds within the chain compared to other bonds in regions close to the chain. In the case of $5 d$ metals, the bonds in the atomic chain, in which all atoms are at the surface, is strengthened due to the relativistic effects of the valence electrons. ${ }^{2,5}$ In contrast, the stabilization of the bond strength in atomic chain made from $3 d$ and $4 d$ metals is not significant, making the fabrication of chains from these metals extremely difficult. Despite these difficulties, however, various interesting properties have been predicted. For example, theoretical studies have shown that the Pd atomic chain is ferromagnetic, even if the length of the atomic chain is short ( $\sim 3$ atom). ${ }^{1}$ Consequently, successful fabrication of atomic chains of $3 d$ and $4 d$ metals, $\mathrm{Pd}$ in particular, is of great scientific interest.

Recently, it was shown that atomic or molecular adsorption on metal surfaces can stabilize the metal atomic contact. $2 \mathrm{~nm}$ long Ag atomic chains were created in the presence of oxygen in UHV at $4 \mathrm{~K}$, while clean $\mathrm{Ag}$ only forms short chains. ${ }^{6}$ In the case of Pd, Pd atomic chains were stabilized under the hydrogen evolution reaction in solution. ${ }^{7}$ In UHV, the conductance of the Pd atomic contact decreased from $2 G_{0} \quad\left(G_{0}=2 e^{2} / h\right.$ to $0.5 G_{0}$ due to the introduction of hydrogen. ${ }^{8}$ While it was revealed that it is possible for the metal atomic chain to be stabilized in the presence of other atoms and molecules, the structure of the stabilized atomic chain is not clear. It is not known whether a molecule adsorbs on the chain surface or if it is incorporated into the chain. Vibrational spectroscopy of a single molecular junction is one promising technique used to investigate the atomic configuration of the single molecular junction. ${ }^{9,10}$ In the present study, we report on the formation of Pd atomic chains in a hydrogen atmosphere. The structure of the atomic chain has been verified by comparing conductance measurements and vibrational spectra of a single molecular junction to those from theoretical calculations.

\section{EXPERIMENTAL}

All measurements were performed using a mechanically controllable break junction (MCBJ) technique (see Ref. 2 for a detailed description). A notched $\mathrm{Pd}(0.1 \mathrm{~mm}$ diameter, $99.99 \%$ purity) wire was glued onto a bending beam and mounted in a three-point bending configuration inside a vacuum chamber. Once under vacuum and cooled to $4.2 \mathrm{~K}$, the Pd wire was broken by mechanical bending of the substrate. The bending was then relaxed to form atomic-sized contacts between the wire ends using a piezo element for fine adjustment. $\mathrm{H}_{2}$ was injected into the chamber via a capillary. dc two-point voltage-biased conductance measurements were performed by applying a voltage in the range from 10 to 150 $\mathrm{mV}$. ac voltage bias conductance measurements were performed using a standard lock-in technique. The conductance was recorded for a fixed contact configuration using a $1 \mathrm{mV}$ ac modulation at a frequency of $7.777 \mathrm{kHz}$ while slowly ramping the dc bias between -100 and $+100 \mathrm{mV}$.

\section{EXPERIMENTAL RESULTS}

Figure 1(a) shows typical conductance traces for clean Pd contacts and for Pd contacts in a $\mathrm{H}_{2}$ atmosphere, recorded with a bias voltage of $0.1 \mathrm{~V}$. For the clean Pd contacts, the conductance decreased in a stepwise fashion and the corresponding conductance histogram [Fig. 1(b)] showed a peak near $2 G_{0}$, which corresponds to a clean Pd atomic contact. After the introduction of $\mathrm{H}_{2}$, a plateau below $1 G_{0}$ was frequently observed and the corresponding histogram [Fig. $1(\mathrm{~b})]$ showed a peak around $0.4 G_{0}$. The obtained conductance histogram agreed with the previously reported results. ${ }^{8}$ Here it should be noticed that the Pd atomic contact in a $\mathrm{H}_{2}$ atmosphere could be stretched to quite long lengths $(\sim 0.5 \mathrm{~nm})$, which suggested the formation of an atomic chain. In order to investigate the chain formation, the length histogram was investigated for the last plateau. Figure 2(a) shows the length histogram of the last conductance plateau for the Pd contacts in a $\mathrm{H}_{2}$ atmosphere, together with that for the clean Pd contacts. The length of the last plateau was taken as the distance between the points at which the con- 

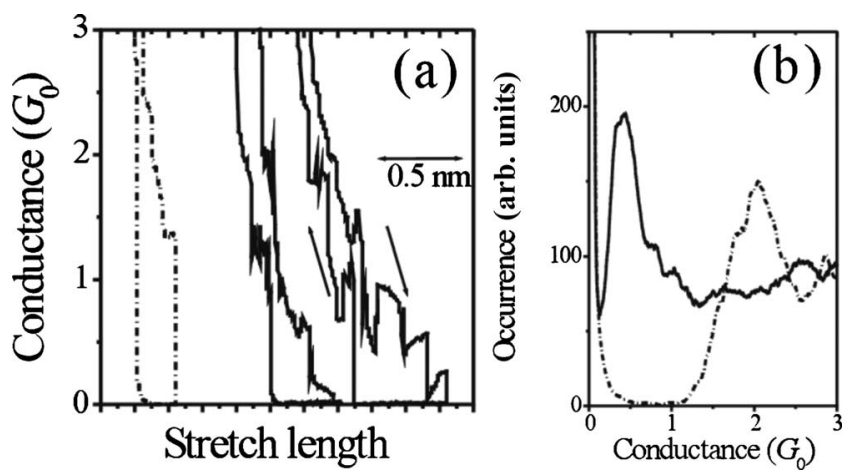

FIG. 1. (a) Breaking and return conductance traces for clean Pd contacts (dotted line) and for $\mathrm{Pd}$ contacts in $\mathrm{a}_{2}$ atmosphere (line). (b) Conductance histograms for the clean Pd contacts (dotted line) and for $\mathrm{Pd}$ contacts in $\mathrm{H}_{2}$ atmosphere (line). Each conductance histogram was constructed from 1000 conductance traces recorded with a bias voltage of $0.1 \mathrm{~V}$ during the breaking of the contact.

ductance dropped below $1.0 G_{0}$ and $0.05 G_{0}$ for the Pd contact in a $\mathrm{H}_{2}$ atmosphere, and the boundaries were $2.5 G_{0}$ and $1.0 G_{0}$ for the clean Pd contact. The length histogram for the $\mathrm{Pd}$ contacts in a $\mathrm{H}_{2}$ atmosphere did not change when the upper boundary was changed from $1.0 G_{0}$ to $2.5 G_{0}$. The length histogram showed that the $\mathrm{Pd}$ atomic contact could be stretched as long as $0.5 \mathrm{~nm}$ in $\mathrm{H}_{2}$ atmosphere, while the clean Pd contact broke within $0.3 \mathrm{~nm}$. Since the Pd-Pd distance is $0.27 \mathrm{~nm}$ for bulk Pd, the final 0.5 -nm-long plateau corresponds to an atomic chain of approximately two Pd atoms.

The formation of the Pd atomic chain was supported by the analysis of the conductance trace. Figure 2(b) shows the average return lengths as a function of chain length, recorded with a bias voltage of $0.1 \mathrm{~V}$. The return length was defined as the distance over which the two electrodes were moved back to make contact after the junction broke. The return length was averaged over 2000 breaking cycles. Apart from an offset of $0.1 \mathrm{~nm}$ due to the elastic response of the banks, the average return length was approximately proportional to the chain length, suggesting that a fragile structure was formed with a length corresponding to that of the last plateau. ${ }^{2,4,5}$ The long plateau over $0.5 \mathrm{~nm}$ length and the one by one relationship between the chain and return length indicated
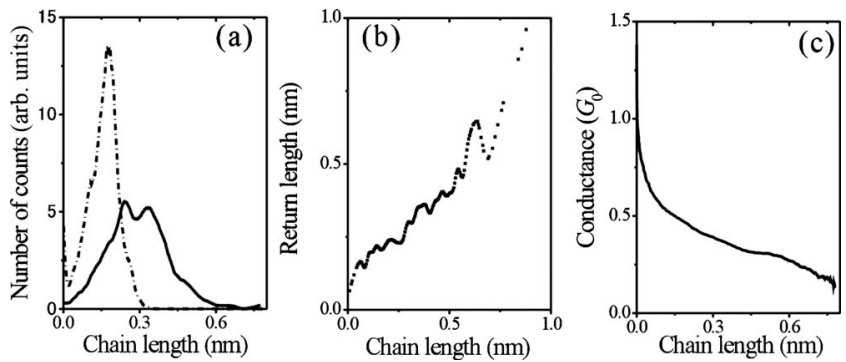

FIG. 2. (a) Length histogram for clean Pd contacts (dotted line) and $\mathrm{Pd}$ contacts in $\mathrm{a}_{2}$ atmosphere (line). (b) Average return lengths as a function of chain length. (c) Average conductance as a function of chain length. Each curve in (a)-(c) was constructed from 2000 conductance traces recorded with a bias voltage of 0.1 $\mathrm{V}$ during the breaking of the contact. that a $\mathrm{Pd}$ atomic chain could be formed in a $\mathrm{H}_{2}$ atmosphere. Figure 2(c) shows the average conductance of the atomic chain as a function of the chain length. The curve was obtained by adding all measured conductance traces from the start value $\left(1 G_{0}\right)$ onward and dividing each length by the number of traces included at that point. It was observed that the mean conductance of the atomic chain decreased with increasing chain length.

In order to investigate the atomic configuration of the atomic chain, the vibrational spectroscopy of a single molecular junction was measured for the Pd atomic contacts in a $\mathrm{H}_{2}$ atmosphere. Figure 3(a) shows examples of the $d^{2} I / d V^{2}$ curves (vibrational spectroscopy of a single molecular junction) for the Pd atomic contacts in a $\mathrm{H}_{2}$ atmosphere taken at a zero bias conductance of $0.15 \sim 0.6 G_{0}$. The spectral shape and energy of the vibrational modes varied with the contacts. Peaks and dips were observed in the spectra depending on the conductance of the contacts. When the conductance of the contact was lower than $0.4 G_{0}$, only peaks were observed in the spectra. Conversely, both peaks and dips were observed in the spectra when the conductance of the contact was larger than $0.4 G_{0}$.

The peaks in the spectra indicated a conductance enhancement above a certain voltage, while the dips indicated conductance suppression. The conductance enhancement can be explained by the opening of an additional tunneling channel for electrons that lose energy to vibrational modes. ${ }^{9-12}$ Likewise, the conductance suppression could be explained as the backscattering of electrons that lose energy to vibrational modes in the ballistic contacts in which the electron transmission probability is close to one. When a molecule symmetrically couples to both metal electrodes and the number of the conduction channel is one, the theoretical investigation predicts that the conductance of the contact would be enhanced by phonon excitation for contacts having a conductance below $0.5 G_{0}$, while the conductance would be suppressed for the contacts having a conductance above $0.5 G_{0}{ }^{11,12}$ The experimental result that only peaks were observed for the contacts having conductance below $0.4 G_{0}$ agrees with this theoretical prediction. Furthermore, the results that both peaks and dips were observed for contacts with conductances above $0.4 G_{0}$, indicate the presence of more than one conductance channel. In the latter case, the presence of more than one channel makes it difficult to discuss the direction of the conductance change induced by the phonon excitation. ${ }^{11,12}$

Our observations indicate that the Pd atoms bridge between the Pd electrodes for contacts with conductances above $0.4 G_{0}$, as discussed below. If a hydrogen bridges the gap between the Pd electrodes, electrons would be transmitted through $1 s$ atomic orbital (in the case of a hydrogen atom bridge) or bonding or antibonding molecular orbitals (in the case of a hydrogen molecule bridge) which would be modulated by Pd. Since these orbitals are not degenerate and are energetically well separated, only one orbital can contribute to the electron transport through the hydrogen atom or molecule bridge. Since the present experimental results suggested multiple conduction channels, hydrogen cannot have formed a bridge between the Pd electrodes as electrons would have been transmitted through $\mathrm{Pd} d$ orbitals. 

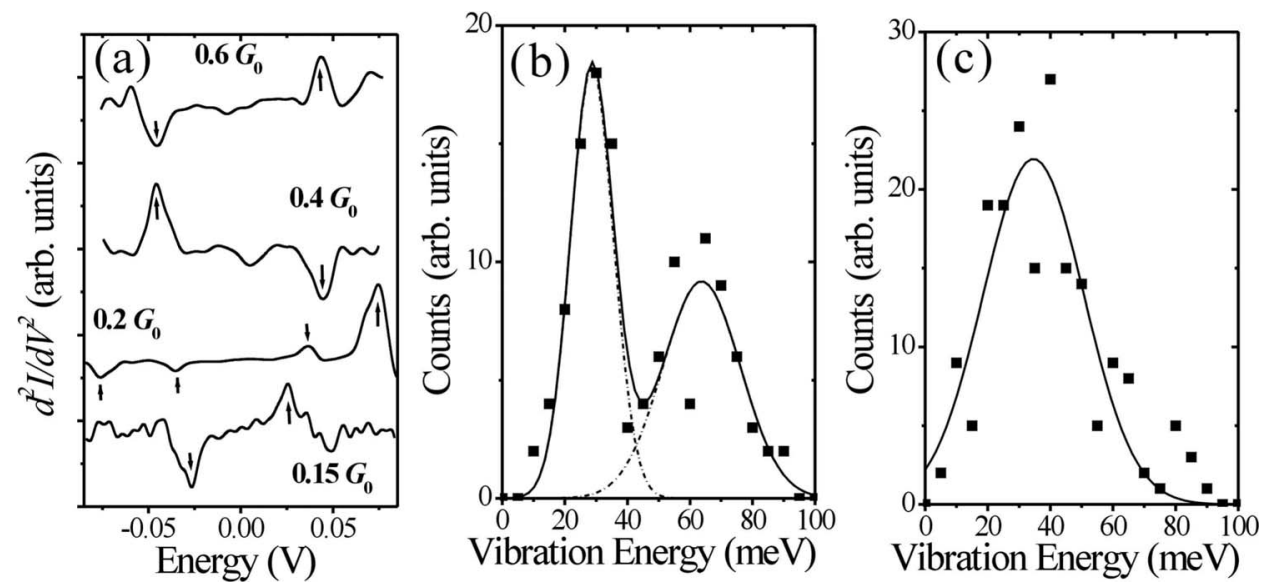

FIG. 3. (a) Typical $d^{2} I / d V^{2}$ curves for Pd contacts in a $\mathrm{H}_{2}$ atmosphere taken at conductance ranging from $0.15 \sim 0.6 G_{0}$. (b) The distribution of vibrational energy for the contacts with conductance below $0.3 G_{0}$. (c) The distribution of vibrational energy for contacts with conductance ranging from $0.3 \sim 0.6 G_{0}$.

In the spectra of Fig. 3, the vibrational modes were observed at $25 \mathrm{meV}$ (conductance of contact: $0.15 G_{0}$ ), 35 and $75 \mathrm{meV}\left(0.2 G_{0}\right), 45 \mathrm{meV}\left(0.4 G_{0}\right)$ and $45 \mathrm{meV}\left(0.6 G_{0}\right)$. While the energy of the vibrational modes varied with the contacts, the distribution shows some defining characteristics. Specifically, it depended on the conductance value of the contacts. The distribution of the vibrational energy of the contacts having conductance below $0.3 G_{0}$ was different from that above $0.3 G_{0}$. Figures 3(b) and 3(c) show the distribution of vibrational energy for contacts having conductances below $0.3 G_{0}$ and $0.3 \sim 0.6 G_{0}$, respectively. The histograms were obtained from 250 spectra of different contacts. The histogram showed two peaks at $29 \mathrm{meV}$ (14 meV in width) and $64 \mathrm{meV}$ ( $25 \mathrm{meV}$ in width) for contacts with conductance below $0.3 G_{0}$ and broad single peak at $35 \mathrm{meV}(32 \mathrm{meV}$ in width) for contacts with conductance of $0.3 \sim 0.6 G_{0}$. Since the conductance and vibrational energy of the atomic contacts is strongly correlated with their atomic configurations, the present results indicate that atomic chains with two different atomic configurations would be formed during stretching of the Pd contacts in $\mathrm{H}_{2}$ atmosphere. The conductance of the atomic chain decreased with the chain length, as shown in Figs. 1(a) and 2(c). The poorly conductive long atomic chain shows two vibrational modes around 29 and $64 \mathrm{meV}$, while the highly conductive short atomic chain shows one vibrational mode around $35 \mathrm{meV}$. Here it should be noted that the spectra for the contact of $0.2 G_{0}$ [Fig. 3(a)] shows two vibrational modes, indicating the presences of two vibrational modes in the contact. The double peaks in the histogram did not, however, originate from the two atomic contacts with different vibrational modes and similar conductance values. Briefly, we comment on the features of the conductance histogram. While the vibrational spectra suggest the formation of atomic chains with two different atomic configurations, the conductance histogram shows a single feature around $0.4 G_{0}$. We think that single feature in the conductance histogram is composed of two broad peaks. The formation of atomic chains with two different atomic configurations is supported by the conductance trace. The last curve in Fig. 1(a) shows steps around $0.5 G_{0}$ and $0.2 G_{0}$.

\section{CALCULATION RESULTS}

To gain insight regarding the structural and vibrational modes of our Pd chain in a $\mathrm{H}_{2}$ atmosphere, we performed density-functional theory (DFT) calculations using the GAUSSIAN03 program. ${ }^{13}$ The hybrid DFT approach, B3LYP, ${ }^{14}$ was employed with the Stuttgart-Koln effective core potentials for Pd (Ref. 15) and Dunning's cc-pVDZ basis set for $\mathrm{H} .{ }^{16}$ The atomic contact part was modeled using two types of clusters, (a) $\mathrm{Pd}_{4}-\mathrm{Pd}-\mathrm{Pd}_{4}$ and (b) $\mathrm{Pd}_{4}-\mathrm{Pd}_{4}$, where a hydrogen molecule was introduced in the area around the central $\mathrm{Pd}$ atoms. The geometry was fully optimized for the respective models and normal mode analyses were performed for the obtained structures. Through preliminary calculations, we have found that $\mathrm{H}_{2}$ cannot attach to the central part of $\mathrm{Pd}$ chains as a molecule, and easily dissociates to hydrogen atoms on the Pd cluster. Thus, geometry optimizations were performed for the respective model clusters with the adsorption of two hydrogen atoms and we succeeded in locating minimum energy structures with no imaginary frequencies for both model clusters.

Figure 4 shows the vibrational modes containing the movement of hydrogen atoms calculated for the optimized structures of (a) $\mathrm{Pd}_{4}-\mathrm{H}-\mathrm{Pd}-\mathrm{H}-\mathrm{Pd}_{4}$ (referred to as "short $\mathrm{Pd}$ chain") and (b) $\mathrm{Pd}_{3}-\mathrm{Pd}-\mathrm{H}-\mathrm{H}-\mathrm{Pd}-\mathrm{Pd}_{3}$ (referred to as "long $\mathrm{Pd}$ chain"). The $\mathrm{H}_{2}$ molecule dissociates to $\mathrm{H}$ atoms in both structures $\left[r_{H H} \sim 0.192 \mathrm{~nm}\right.$ in structure (b)] which work to stabilize the contact part of the Pd chain. The present calculations reveal that the short hydrogen-adsorbed shows the five vibrational modes of the Pd chain around $23-31 \mathrm{meV}$, while the long hydrogen incorporated Pd contact shows the vibrational modes at 41 and $59 \mathrm{meV}$.

The vibrational modes observed in the present experiments are discussed based on these calculation results and previously reported results of nanocontacts. Pd nanocontact and $\mathrm{Pd}$ nanocontact in a $\mathrm{H}_{2}$ atmosphere were investigated using point contact spectroscopy. In these experiments, $\mathrm{Pd}$ crystal vibrational modes were observed around $15-20 \mathrm{meV}$ for the Pd nanocontact and, likewise, the vibrational modes of dissolved $\mathrm{H}$ atoms was observed around $60 \mathrm{meV}$ for con- 
(a) Short Pd chain

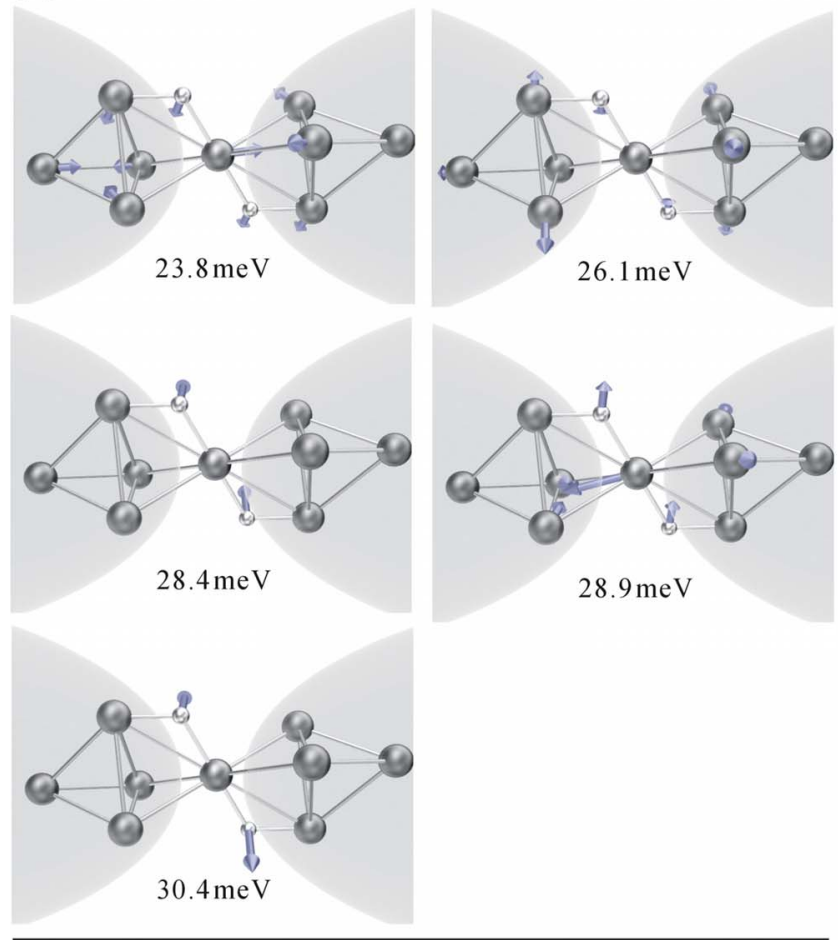

(b) Long Pd chain
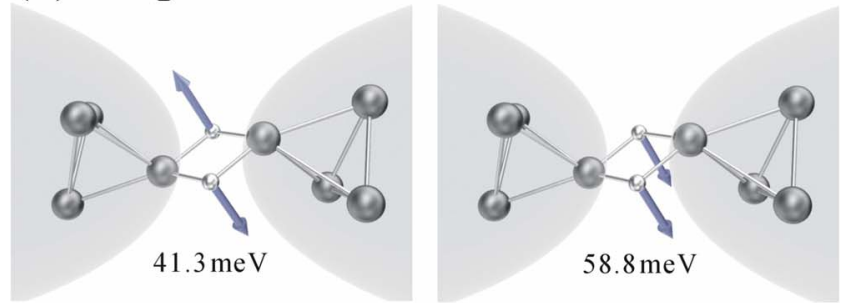

FIG. 4. (Color online) Vibrational modes containing the movement of hydrogen atoms calculated for the optimized structures of (a) $\mathrm{Pd}_{4}-\mathrm{H}-\mathrm{Pd}-\mathrm{H}-\mathrm{Pd}_{4}$ (short $\mathrm{Pd}$ chain) and (b) $\mathrm{Pd}_{3}-\mathrm{Pd}-\mathrm{H}-\mathrm{H}-\mathrm{Pd}-\mathrm{Pd}_{3}$ (long Pd chain). The length of the arrows indicates the amplitude of the modes.

tacts in a $\mathrm{H}_{2}$ atmosphere. In the present study, the histogram of the vibrational modes showed two peaks around 29 and 64 $\mathrm{meV}$ for the long atomic chain, and a broad single peak around $35 \mathrm{meV}$ for the short atomic chain. Based on these experimental and theoretical calculation results, an experimentally obtained broad single peak around $35 \mathrm{meV}$ for the short atomic chain would correspond to the 5 calculated vibrational modes ranging from $23-31 \mathrm{meV}$. Since energy differences among the five modes are small, they appear as a single broad peak in the histogram. $35 \mathrm{meV}$ is close to the energy of the vibrational mode of the Pd crystal (15-20 $\mathrm{meV}$ ) and the vibrational modes of Pd in the stem parts of the electrodes might contribute to the $35 \mathrm{meV}$ peak. The two experimentally obtained peaks around 29 and $64 \mathrm{meV}$ for the long atomic chains correspond to the calculated vibrational modes at 41 and $59 \mathrm{meV}$. For the long atomic chains, the vibrational modes of Pd in the stem part of the electrodes and the modes of dissolves $\mathrm{H}$ atoms in the Pd crystal can contribute to the peaks around 29 and $64 \mathrm{meV}$, respectively. In both short and long atomic chains, the peaks in the histogram of the vibrational modes were broad. The atomic chains whose atomic configuration was slightly modified from the stable structure could be formed under the present experimental conditions, and thus, the vibrational modes from all of these structures can contribute to the peaks, causing them to broaden. Here, we briefly comment on the short atomic chains. As discussed before, the vibrational spectra suggest that electrons can be transmitted through the Pd rather than the hydrogen for contacts with conductance above $0.4 G_{0}$ (short Pd chain), which agreed with the theoretical calculation results.

The conductance of the Pd atomic contacts with similar atomic configurations has been investigated using firstprinciple molecular dynamics simulations. ${ }^{17}$ The conductance values of short and long Pd chains are reported as $1 G_{0}$ and $0.5 G_{0}$, respectively. It was found that the conductance of the short Pd chain is smaller than that of the long Pd chain. Our experimental results show atomic chain conductance decreased with chain length, as shown in Figs. 1(a) and 2(c), which is in agreement with the results of theoretical calculations. The smaller conductance values obtained in the experimental results can be explained as arising from the adsorption or incorporation of hydrogen onto the stems of the $\mathrm{Pd}$ electrodes since hydrogen molecules may dissociate and migrate into the bulk Pd. The theoretical calculations show that the conductances of the hydrogen-adsorbed and hydrogen incorporated Pd contacts decreased by $30 \sim 60 \%$ depending on the amount of the hydrogen in the Pd electrodes. ${ }^{18}$ Our experimental and calculated results combined with previous theoretical simulations ${ }^{17}$ revealed that the atomic chain of two Pd atoms in contact with the hydrogen bridge can be formed when a Pd contact is stretched in a $\mathrm{H}_{2}$ atmosphere while clean contacts do not, in fact, form an atomic chain.

\section{CONCLUSIONS}

We have investigated the atomic configuration and conductance of Pd atomic contacts in $\mathrm{H}_{2}$ atmosphere. The formation of Pd atomic chains were demonstrated using the length histogram of the last conductance plateau. Vibrational spectroscopy and the results of theoretical calculations show the formation of highly conductive short hydrogen-adsorbed $\mathrm{Pd}$ chain and low conductive long hydrogen incorporated Pd contacts.

\section{ACKNOWLEDGMENTS}

This work was supported by a Grant-in-Aid for Scientific Research on Priority Areas "Electron transport through a linked molecule in nanoscale," Effective Utilization of Elements "Nano-Hybridized Precious-Metal-Free Catalysts for Chemical Energy Conversion," and the Global COE Program (Grant No. B01) from MEXT. 
*Present address: Department of Chemistry, Graduate School of Science and Engineering, Tokyo Institute of Technology 2-12-1 W4-10 Ookayama, Meguro-ku, Tokyo 152-8551, Japan; kiguti@chem.titech.ac.jp

${ }^{1}$ A. Delin, E. Tosatti, and R. Weht, Phys. Rev. Lett. 92, 057201 (2004).

${ }^{2}$ N. Agrait, A. L. Yeyati, and J. M. van Ruitenbeek, Phys. Rep. 377, 81 (2003).

${ }^{3}$ H. Ohnishi, Y. Kondo, and K. Takayanagi, Nature (London) 395, 780 (1998).

${ }^{4}$ A. I. Yanson, G. R. Bollinger, H. E. van den Brom, N. Agraït, and J. M. van Ruitenbeek, Nature (London) 395, 783 (1998).

${ }^{5}$ R. H. M. Smit, C. Untiedt, A. I. Yanson, and J. M. van Ruitenbeek, Phys. Rev. Lett. 87, 266102 (2001).

${ }^{6}$ W. H. A. Thijssen, D. Marjenburgh, R. H. Bremmer, and J. M. van Ruitenbeek, Phys. Rev. Lett. 96, 026806 (2006).

${ }^{7}$ M. Kiguchi and K. Murakoshi, Appl. Phys. Lett. 88, 253112 (2006).

${ }^{8}$ Sz. Csonka, A. Halbritter, G. Mihaly, O. I. Shklyarevskii, S. Speller, and H. van Kempen, Phys. Rev. Lett. 93, 016802
(2004).

${ }^{9}$ R. H. M. Smit, Y. Noat, C. Untiedt, N. D. Lang, M. C. van Hemert, and J. M. van Ruitenbeek, Nature (London) 419, 906 (2002).

${ }^{10}$ M. Kiguchi, O. Tal, S. Wohlthat, F. Pauly, M. Krieger, D. Djukic, J. C. Cuevas, and J. M. van Ruitenbeek, Phys. Rev. Lett. 101, 046801 (2008).

${ }^{11}$ T. Shimazaki and Y. Asai, Phys. Rev. B 77, 115428 (2008).

${ }^{12}$ M. Paulsson, T. Frederiksen, H. Ueba, N. Lorente, and M. Brandbyge, Phys. Rev. Lett. 100, 226604 (2008).

${ }^{13}$ M. J. Frisch et al., GAUSSIAN03, Revision C.02, Gaussian, Inc., Wallingford CT, 2004.

${ }^{14}$ A. D. Becke, J. Chem. Phys. 98, 5648 (1993).

${ }^{15}$ D. Andrae, U. Hausermann, M. Dolg, H. Stoll, and H. Preus, Theor. Chim. Acta 77, 123 (1990).

${ }^{16}$ Thom H. Dunning, J. Chem. Phys. 90, 1007 (1989).

${ }^{17}$ B. Pieczyrak, C. Gonzalez, P. Jelnek, R. Perez, J. Ortega, and F. Flores, Nanotechnology 19, 335711 (2008).

${ }^{18}$ X. Wu, Q. Li, and J. Yang, Phys. Rev. B 72, 115438 (2005). 